experience and evaluate the clinical efficacy and safety of removable SEMS.

Methods We conducted a retrospective study between January 2005 and July 2009. Data analysis was carried out using SPSS.

Results 36 stents were inserted in a total of 33 patients (20 males and 13 females). The mean age was $59.7 \pm 14.4$ years (range $22-81$ ). 32 stents (84.2\%) were deployed for malignant conditions and 4 $(10.5 \%)$ for benign diseases. $81.8 \%$ of patients had malignant airway obstruction and $6.1 \%$ had tracheo-oesophageal fistulae. Obstruction was located to the trachea in $44.4 \%$ of cases. $88.9 \%$ of patients reported improvement in symptoms in the first $24 \mathrm{~h} .62 .9 \%$ of patients were in severe respiratory distress prior to SEMS insertion, with immediate improvement thereafter. Complications were reported in 14 stents, with 2 presenting within $24 \mathrm{~h}$. We recorded 1 case of stent fracture, 6 cases of migration, 5 cases of mucus plugging, 1 minor bronchial tear associated with stent placement, and 1 case of stent-associated respiratory tract infection. 12 stents were easily removed, with no associated complications. 17 deaths were reported, none related to stent insertion and all were in patients with advanced malignant disease. Survival for these patients ranged from 7 to 430 days poststent insertion.

Conclusions SEMS provide an effective, safe and feasible therapeutic modality in patients with benign and malignant airway stenoses. Although associated with significant complications, they have the advantage of providing immediate relief from breathlessness and imminent asphyxia. In patients with malignancy, SEMS enable definitive oncological treatment to be given electively, and in greater safety, and are easily removed after completion of treatment.

\section{COPD: cells and genes}

\section{S105 FIBRINOPEPTIDE Aa360: A F00TPRINT OF NEUTROPHIL ELASTASE ACTIVITY}

${ }^{1} \mathrm{R}$ Carter, ${ }^{2} \mathrm{R}$ Mumford, ${ }^{2} \mathrm{~K}$ Treonze, ${ }^{1} \mathrm{R}$ Stockley. ${ }^{1}$ University Hospital Birmingham, Birmingham, UK; ${ }^{2} \mathrm{MSD}$, Rahway, USA

\section{doi:10.1136/thx.2009.127092a}

Initial descriptions of the association between $\alpha$-1-antitrypsin deficiency (AATD) and lower zone pulmonary emphysema ${ }^{1}$ were closely followed by the confirmation that neutrophil elastase (NE) could induce emphysema in animal models. ${ }^{2}$ Mathematical and in vitro models have demonstrated an exponential relationship between released enzyme activity and distance from the neutrophil ${ }^{34}$ and since the neutrophil NE concentration at release is substantially higher than the physiological concentration of AAT an area of obligate proteolytic damage will occur, which is substantially larger in patients with AATD. This process of quantum proteolysis is a simple concept that explains the increased susceptibility of subjects with AATD to emphysema, but, since it occurs in the neutrophil microenvironment, it has been difficult to demonstrate in vivo. The aim of this study was to explore the relationship of a specific (preinhibition) NE cleavage product of fibrinogen (Aa360) to AAT and the presence of airflow obstruction in deficient subjects.

Methods Plasma A $\alpha 360$ was measured in a group of 68 subjects with a wide range of plasma AAT levels. Subsequently, postbronchodilator spirometry was related to plasma A $\alpha 360$ concentrations in a further 72 patients ( 49 male and 23 female) with PiZ AATD in the stable state. Correlations were determined using non-parametric statistical tests.

Results Aa360 showed an exponential relationship to plasma AAT concentration that increased at levels $<11 \mu \mathrm{m}$ (the putative protective threshold), as shown in fig 1, consistent with theoretical modelling and in vitro experiments. The mean forced expiratory

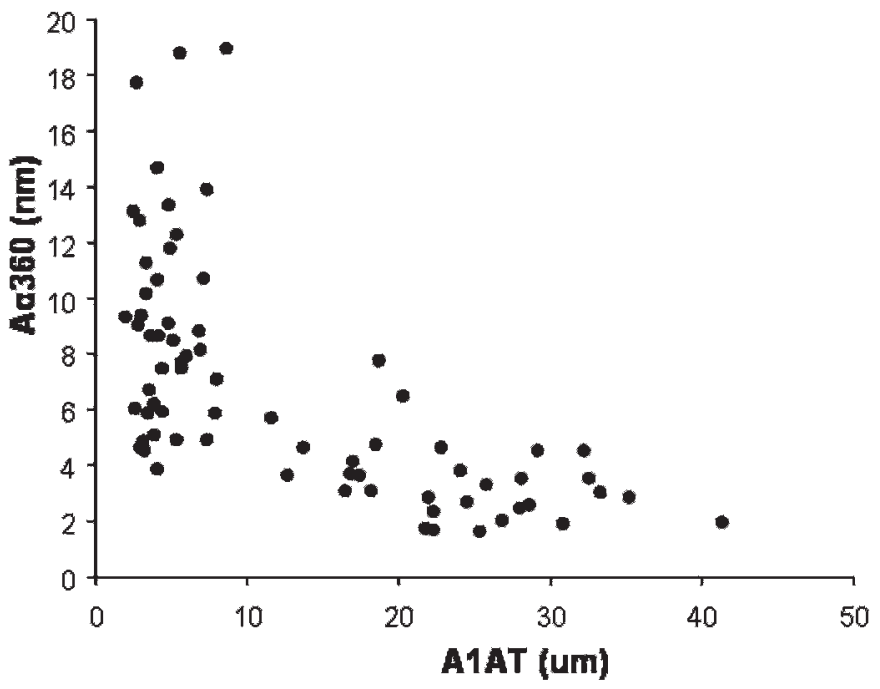

The exponential relationship between AlAT and Aa360

Abstract S105 Figure 1. A1AT, $\alpha$-1-antitrypsin.

volume in $1 \mathrm{~s}\left(\mathrm{FEV}_{1}\right)$ and $\mathrm{FEV}$-:vital capacity (VC) were $50.7 \%$ predicted and $37.26 \%$, respectively, and 61 patients had an $\mathrm{FEV}_{1}: \mathrm{VC}$ $<70 \%$. There was no relationship between A 2360 and age, height, gender or smoking status. However, A 2360 correlated with $\mathrm{FEV}_{1}$ $(p=0.01), F_{1}: \mathrm{VC}(p=0.008)$ and residual volume $(p=0.013)$.

Conclusion The Aa360 concentration is a direct measure of the destructive activity of neutrophils, relates (as predicted) to the AAT level and correlates with spirometric markers of severity in patients with AATD.

1. Clin Radiol 1992:45:260-6.

2. Am Rev Respir Dis 1985;132:362-6.

3. Biochemistry 1995;34:16171-7.

4. J Immunol 1996;157:2624-31.

\section{S106 DIESEL EXHAUST PARTICLES POTENTIATE INFLAMMATION AND RENDER IT RESISTANT TO IL-1 ANTAGONISM IN VITRO}

${ }^{1} \mathrm{~N}$ Chaudhuri, ${ }^{1} \mathrm{LC}$ Parker, ${ }^{1} \mathrm{I}$ Sabroe, ${ }^{2 \mathrm{~K}}$ Donaldson. ${ }^{1}$ Academic Unit of Respiratory Medicine, School of Medicine and Biomedical Sciences, University of Sheffield, Sheffield, UK; ' 2 Queens Medical Research Unit, Edinburgh, UK

\section{doi:10.1136/thx.2009.127092b}

Introduction and Objectives Inhalation of small particulate matter is a major cause of airway inflammation, with fragments of bacteria, viruses and various environmental pollutants causing inflammation and potentiation of human respiratory diseases such as chronic obstructive pulmonary disease (COPD) and asthma. Diesel exhaust particles (DEPs) are the major component of air pollution. The influence of DEPs on the interactions between resident epithelial cells and infiltrating leucocytes remains unexplored. In this study we investigated the potential for DEPs to induce inflammation in the airway, and subsequently explored its capacity to modulate the inflammatory response to pathogenic stimuli, a situation that will commonly occur during inhalation of environmental particulates and microbial products.

Methods The effects of DEPs were studied in co-cultures of primary human monocytes and the immortalised bronchial epithelial cell line, BEAS-2B. Co-cultures, or monoculture controls, were treated with DEPs in the presence or absence of Toll-like receptor 4 (TLR4) and TLR5 agonists (lipopolysaccharide (LPS) and flagellin, respectively) and an interleukin-1 (IL-1) receptor antagonist (IL-1ra). Changes in cytokine release were measured by ELISA. 
Results We demonstrated that DEPs dose-dependently induced CXCL8 release in our co-culture of BEAS-2B cells and monocytes, although this was a modest response when DEPs were administered alone. Notably we found that DEPs accentuated the cellular response to minimal amounts of TLR agonists, which in their own right had only modest actions in our co-culture system. Our previous studies have revealed the existence of IL-1-dependent inflammatory networks potentially amenable to therapeutic targeting. Here we have shown that LPS co-culture responses are blocked by inhibition of IL-1. However, in the presence of DEPs, IL1 antagonism no longer suppressed activation of co-cultures by LPS. This was paralleled with a decrease in extracellular IL-1 with no alteration of intracellular IL-1 as measured by western blot analysis, thus highlighting an uncoupling between IL-1 production and release in the presence of DEPs. We have demonstrated a possible mechanism for this disruption of IL-1 release in co-cultures by DEPs' ability to sequester exogenous ATP, a key molecule involved in IL-1 processing and release.

Conclusions Our data reveal a novel mechanism of DEP toxicity, with its principal action the dramatic accentuation of the proinflammatory properties of very small amounts of TLR agonists. These effects may enhance the inflammatory potential of environmental pollutants through their synergistic actions and, coupled with the switch to an IL-1-independent system not targeted by natural IL-1ra, reveal how inflammation might be initiated and perpetuated by diesel exhaust nanoparticles.

\section{S107 INHALED CORTICOSTEROIDS IN AIRWAY REMODELLING IN COPD}

A Soltani, S Sohal, D Reid, HK Muller, S Weston, R Wood-Baker, EH Walters. Menzies Research Institute, University of Tasmania, Hobart, Australia

doi:10.1136/thx.2009.127092c

Introduction and Objectives Both chronic obstructive pulmonary disease (COPD) and asthma are chronic inflammatory diseases of airways. Reticular basement membrane (RBM) has been reported to be thicker and mucosal vessels increased in the airways in asthma; and both these changes responded to inhaled corticosteroids (ICS). Knowledge about the effects of ICS on airway remodelling in COPD is limited. We have already reported that the RBM is fragmented, vessels in the RBM are increased (fig 1) and vessels in the lamina propria are reduced in COPD (European Respiratory Society Meeting, Vienna, September 2009). We hypothesised that the $\mathrm{RBM}$ and vascular remodelling in COPD will also respond to treatment with ICS.

Methods This was a double-blind, randomised clinical trial comparing the effects of ICS with placebo using bronchial biopsies from subjects with COPD. Perbronchoscopic biopsies from subjects with COPD were stained with collagen IV antibody to detect the basement membrane of vessels (and corroborated with anti-von Willebrand factor). Splits in the RBM, and number and area of vessels in the RBM and lamina propria were measured (fig 1) and compared before and after treatment.

Results 34 subjects were randomly allocated 2:1 to active treatment (fluticasone propionate $500 \mu \mathrm{g}$ twice daily) or placebo. There were no significant differences between the groups in demographics (female/male $9 / 14$ vs $4 / 7$, current smokers/ex-smokers $13 / 10$ vs 4/7, median (range) for age: 61 (49-69) vs 61 (52-69) years, pack-year smoking history: 44 (18-151) vs 51 (22-148) and forced expiratory volume in $1 \mathrm{~s}\left(\mathrm{FEV}_{1}\right) \%$ predicted). RBM splitting and vessel distribution were not significantly different between the groups before treatment, although both were highly abnormal. Splitting responded to treatment with ICS (median (range) 19.1 (0.2-42.8) before vs $2.6(0-88.6) \mu \mathrm{m} / \mu \mathrm{m} \mathrm{RBM} \times 100$ after ICS, $\mathrm{p}<0.03)$ but did

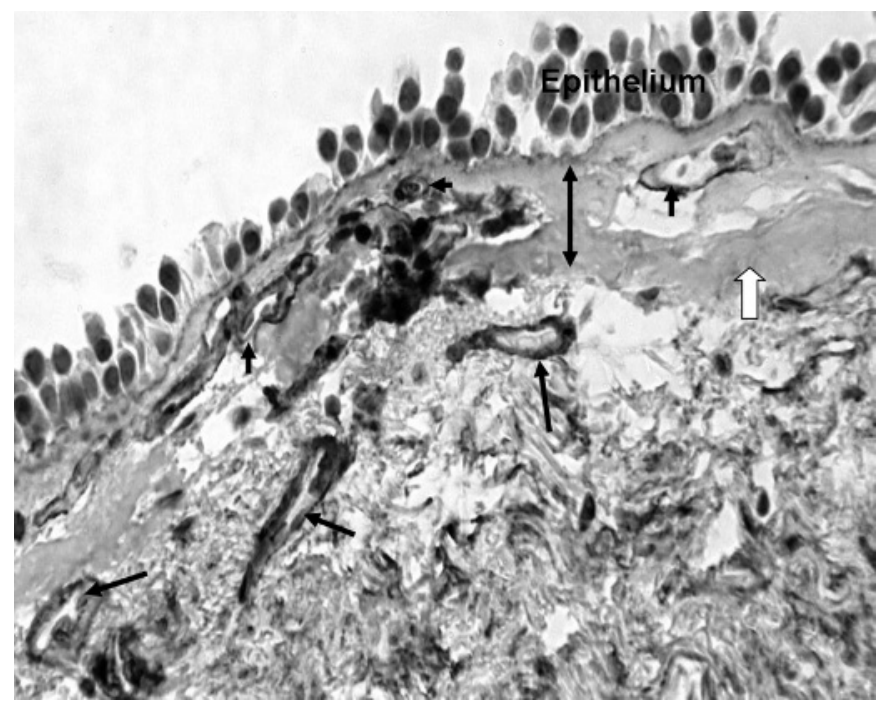

Abstract S107 Figure 1 Reticular basement membrane (RBM) (double-headed arrow) is fragmented. Splitting is marked with a white arrow. Vessels in the lamina propria and RBM are marked with black arrows. (collagen IV antibody, $\times 400)$.

not change with placebo (24 (6.6-109) before vs $26.9(2.5-48.5) \mu \mathrm{m} /$ $\mu \mathrm{m}$ RBM $\times 100$ after placebo). Vessel number and distribution did not change significantly with ICS.

Conclusions RBM fragmentation in COPD is reversible with ICS, but vessel pathology is not. These findings are compatible with the relative resistance to ICS treatment in COPD compared with asthma.

\section{S108 DECREASED MACROPHAGE FUNCTION IN COPD}

${ }^{1}$ CMR Thomas, ${ }^{2} \mathrm{MA}$ Saunders, ${ }^{3} \mathrm{JA}$ Wedzicha, ${ }^{1} \mathrm{PJ}$ Barnes, ${ }^{1} \mathrm{LE}$ Donnelly. ${ }^{1} / \mathrm{mperial}$ College, London, UK; ${ }^{2}$ AstraZeneca, Loughborough, UK; ${ }^{3}$ Royal Free Hospital, London, UK

\section{doi:10.1136/thx.2009.127092d}

Introduction Acute exacerbations of chronic obstructive pulmonary disease (COPD) are the most common cause of acute medical admission in the UK. Viral and bacterial infections of the lung are causative in the majority of exacerbations. Alveolar macrophages (AMs) are essential for clearance of inhaled particles, including pathogens. Therefore, a reduction in the phagocytic function of AMs may lead to bacterial colonisation and increased frequency of exacerbations.

Methods Monocyte-derived macrophages (MDMs) were used as a model of AMs and were generated from blood monocytes by culture for 12 days in medium containing granulocyte-macrophage colonystimulating factor (GM-CSF). Phagocytosis of fluorescently labelled polystyrene beads, Haemophilus influenzae (HI) or Streptococcus pneumoniae (SP) was measured by fluorimetry. Release of tumour necrosis factor $\alpha(\mathrm{TNF} \alpha)$ from $4 \mathrm{~h}$ MDM supernatants was measured using ELISA.

Results MDMs from subjects with COPD showed equivalent phagocytosis of beads compared with MDMs from controls. However, COPD MDMs showed significantly reduced phagocytosis of both bacteria at all time points, which was most apparent after $4 \mathrm{~h}$. COPD MDMs incubated with $\mathrm{HI}$ showed a $32 \%$ reduction in phagocytosis compared with cells from healthy subjects (COPD $11737 \pm 621$ relative fluorescence units (RFU), $n=13$ vs healthy $16426 \pm 1434 \mathrm{RFU}, \mathrm{n}=5, \mathrm{p}<0.01)$. Incubation with SP showed a $52 \%$ reduction in phagocytosis by COPD MDMs compared with 
cells from healthy subjects (COPD $7183 \pm 485$ RFU, $n=13$ vs healthy $13702 \pm 2933$ RFU, $n=5, p<0.01)$. TNF $\alpha$ release at $4 \mathrm{~h}$ was significantly increased from baseline values in all subjects $(p<0.001)$. COPD MDMs released significantly more TNF $\alpha$ than healthy MDMs when incubated with beads $(0.3 \pm 0.07 \mathrm{ng} / \mathrm{ml}$ vs $0.2 \pm 0.04 \mathrm{ng} / \mathrm{ml}, \mathrm{p}<0.01)$. Healthy $\mathrm{MDMs}$ released significantly more TNF $\alpha$ compared with COPD MDMs when incubated with bacteria $(3.8 \pm 0.4 \mathrm{ng} / \mathrm{ml}$ vs $2.1 \pm 0.1 \mathrm{ng} / \mathrm{ml}, \quad \mathrm{p}<0.001$ for $S P$, $19.6 \pm 4.2 \mathrm{ng} / \mathrm{ml}$ vs $9.5 \pm 0.98 \mathrm{ng} / \mathrm{ml}, \mathrm{p}<0.05$ for $\mathrm{HI}$ ).

Discussion These data indicate that macrophages from subjects with COPD show defective phagocytosis of pathogens but not inert particles. This is not associated with an increased inflammatory response at $4 \mathrm{~h}$. Therefore, in COPD, macrophages may be defective in both their phagocytic and inflammatory response to pathogens, which in turn may predispose to bacterial colonisation and increased frequency of exacerbations.

\section{S109 MORPHOMETRIC COMPARISON OF AIRWAY REMODELLING IN CHRONIC OBSTRUCTIVE PULMONARY DISEASE AND OBLITERATIVE BRONCHIOLITIS}

LA Borthwick, CL Pate, LS Mackay, GE Johnson, PA Corris, J Majo, AJ Fisher. Newcastle University, Newcastle-Upon-Tyne, UK

\section{doi:10.1136/thx.2009.127092e}

Introduction Airway remodelling and fibrotic repair in small airways contributes to chronic lung diseases characterised by repeated airway injury. Severe chronic obstructive pulmonary disease (COPD) and post-transplant obliterative bronchiolitis (OB) are both characterised by marked airflow obstruction yet have very different aetiologies. We hypothesised that there may be similarities in the patterns of airway remodelling which suggest a common pathway of dysregulated repair via transforming growth factor $\beta$ (TGF $\beta$ ) in both conditions.

Methods Airways $<2 \mathrm{~mm}$ in diameter from patients with $\mathrm{OB}$ $(n=6)$, severe COPD $(n=6)$ and unused donor lungs as control $(n=3)$ were identified in explanted formalin-fixed lung tissue. The median number of small airways evaluated per case was 5 (range 4 12). The proportion of the airway cross-section consisting of lumen, epithelium and subepithelial layer was measured by morphometry and compared between the three groups. The cellularity of the subepithelial layer was also assessed. The extent of phosphoSMAD2/3 staining as a marker of TGF $\beta$ signalling was assessed using a semi-quantitative scoring system.

Results The subepithelial layer was significantly expanded in $\mathrm{OB}$ airways (mean \pm SEM; $48.7 \pm 20.36 \%$ ) compared with control $(20.6 \pm 8.0 \%)$ and COPD $(26.3 \pm 6.8 \%)$. The cellularity of this expansion varied greatly; some showed a sparsely cellular fibrotic subepithelial layer, whereas others were cellular with a dense inflammatory infiltrate. The epithelial layer was significantly decreased in the $\mathrm{OB}$ airways $(13.4 \pm 11.81 \%)$ and significantly increased in COPD airways (34.9 $\pm 6.8 \%$ ) compared with control $(29.8 \pm 7.75 \%)$. The lumen was significantly decreased in the OB $(17.1 \pm 16.06 \%)$ and COPD airways $(16.3 \pm 8.52 \%)$ compared with control $(36.0 \pm 17.3 \%)$. A significantly higher proportion of $O B$ airways showed strong positive phosphoSMAD 2/3 nuclear staining compared with COPD and control.

Conclusions In COPD the reduction in the area of the lumen is a consequence of an increase in area of the epithelial layer and smooth muscle layer. In $\mathrm{OB}$ the reduction in the area of the lumen is due to an expansion in the subepithelial layer, suggesting that remodelling in this compartment is more critical to the airway obstruction in $\mathrm{OB}$ than in COPD. The increased phosphoSMAD2/3 staining in $\mathrm{OB}$ airways suggests that TGF $\beta$ may play a significant role in impaired epithelial repair and expansion of the subepithelial layer.

\section{S110 AUTOIMMUNITY-ASSOCIATED GENETIC POLYMORPHISMS IN} CHRONIC OBSTRUCTIVE PULMONARY DISEASE

${ }^{1}$ AM Wood, ${ }^{2}$ RA Stockley. ' University of Birmingham, Birmingham, UK; ${ }^{2}$ University Hospitals Birmingham, Birmingham, UK

doi:10.1136/thx.2009.127092f

Background Chronic obstructive pulmonary disease (COPD) has been associated with antielastin antibodies in man, whilst in animal models both antielastin and antiendothelial antibodies have been observed. Furthermore, transfer of $\mathrm{T}$ cells from animals with emphysema and autoantibodies can result in disease in healthy animals. Finally, a lymphocytic infiltrate is observed in the lung, and oligoclonal $\mathrm{T}$ lymphocyte populations have been reported, implying accumulation due to antigenic stimulation. Taken together with our previous work showing human leucocyte antigen (HLA) associations with lung function, this suggests an autoimmune component exists in COPD. We have sought further evidence for this hypothesis at the genetic level.

Methods 428 subjects with the PiZZ genotype of $\alpha$-1-antitrypsin deficiency and a full clinical assessment were genotyped for rs11571297, within the CTLA4 gene, and rs2476601, within the PTPN22 gene, carried out using TaqMan genotyping technologies (ABI, UK). Genetic association with forced expiratory volume in $1 \mathrm{~s}$ $\left(\mathrm{FEV}_{1}\right)$ was assessed by linear regression, and with emphysema by logistic regression, adjusting for age, gender and smoke exposure.

Results The genotyping success rate was $94.5 \%$ and both polymorphisms were in Hardy-Weinberg equilibrium in the population. The $\mathrm{T}$ allele of the CTLA4 polymorphism was associated with better lung function, such that $\mathrm{FEV}_{1}$ was $5 \%$ higher on average in subjects with this allele $(p<0.001)$. This equated to a reduced risk of CT-diagnosed emphysema, with an odds ratio of 0.63 (95\% CI 0.46 to 0.89 ). The PTPN22 polymorphism did not exhibit any associations within the population.

Conclusions The CTLA4 gene lies within a peak seen in a linkage study in COPD, and two polymorphisms within it have recently been associated with chronic bronchitis. Our results add further replication, making it more likely it is a true genetic risk factor. The same polymorphisms have been associated with known autoimmune diseases, thus suggesting that COPD may have an autoimmune component determined in part at the genetic level.

\section{Pathways of vascular remodelling in $\mathrm{PAH}$}

\section{S111 BONE MORPHOGENETIC PROTEIN 9 INCREASES ENDOTHELIN- 1 RELEASE BY HUMAN PULMONARY ARTERY ENDOTHELIAL CELLS VIA A MAPK-DEPENDENT PATHWAY}

${ }^{1} \mathrm{JES}$ Park, ${ }^{2} \mathrm{PD}$ Upton, ${ }^{1} \mathrm{PM}$ de Souza, ${ }^{2} \mathrm{RJ}$ Davies, ${ }^{2} \mathrm{NW}$ Morrell, 'MJD Griffiths, ${ }^{1} \mathrm{SJ}$

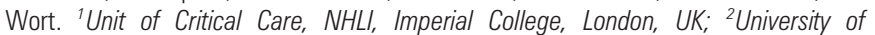
Cambridge School of Clinical Medicine, Addenbrooke's/CUHNHSFT and Papworth Hospitals, Cambridge, UK

\section{doi:10.1136/thx.2009.127092g}

Introduction Mutations in the bone morphogenetic protein (BMP) receptor II (BMPR-II), a member of the transforming growth factor $\beta$ (TGF $\beta$ ) receptor superfamily, are associated with familial pulmonary artery hypertension (PAH), whilst mutations of activin receptor-like kinase-1 (Alk-1) cause hereditary haemorrhagic telangiectasia (HHT). BMP9 binds BMPRII/Alk-1 complex in endothelial cells (ECs) and is reported to be a vascular quiescence factor. ${ }^{1}$ In addition, constitutively active Alk-1 in ECs induces endothelin-1 (ET-1) production, a contributor to vascular remodelling. Dysregulation of these pathways may therefore affect vascular integrity, as seen in both disease processes. We aimed to investigate the effect of BMP9 on ET-1 regulation. 